\title{
Azithromycin in the Treatment of Preterm Prelabor Rupture of Membranes Demonstrates a Lower Risk of Chorioamnionitis and Postpartum Endometritis with an Equivalent Latency Period Compared with Erythromycin Antibiotic Regimens
}

\author{
Daniel Martingano $\mathbb{D}$, ${ }^{1,2}$ Shailini Singh, ${ }^{3,4}$ and Antonina Mitrofanova $\mathbb{D}^{1}$ \\ ${ }^{1}$ Department of Biomedical and Health Informatics, Rutgers University School of Health Professions, Newark, NJ, USA \\ ${ }^{2}$ Department of Obstetrics \& Gynecology, St. John's Episcopal Hospital, Far Rockaway, NY, USA \\ ${ }^{3}$ Division of Maternal-Fetal Medicine, Newark Beth Israel Medical Center, Newark, NJ, USA \\ ${ }^{4}$ Department of Obstetrics \& Gynecology, Rutgers Robert Wood Johnson Medical School, New Brunswick, NJ, USA
}

Correspondence should be addressed to Daniel Martingano; dm1150@shp.rutgers.edu

and Antonina Mitrofanova; amitrofa@shp.rutgers.edu

Received 22 April 2020; Accepted 10 June 2020; Published 9 July 2020

Academic Editor: Bryan Larsen

Copyright (c) 2020 Daniel Martingano et al. This is an open access article distributed under the Creative Commons Attribution License, which permits unrestricted use, distribution, and reproduction in any medium, provided the original work is properly cited.

\begin{abstract}
Objective. To determine if antibiotic regimens including azithromycin versus erythromycin has an impact on pregnancy latency and development of clinical chorioamnionitis in the context of preterm prelabor rupture of membranes. Study Design. We conducted a prospective observational cohort study and followed all women receiving antibiotic regimens including either azithromycin or erythromycin in the context of preterm prelabor rupture of membranes. Primary outcomes were the duration of pregnancy latency period and development of chorioamnionitis. Secondary outcomes included neonatal sepsis with positive blood culture, cesarean delivery, postpartum endometritis, and meconium-stained amniotic fluid. Results. This study included 310 patients, with 142 receiving the azithromycin regimen and 168 receiving the erythromycin regimen. Patients receiving the azithromycin regimen had a statistically significant advantage in overall rates of clinical chorioamnionitis $(13.4 \%$ versus $25 \%$, $p=0.010)$, neonatal sepsis $(4.9 \%$ versus $14.9 \%, p=0.004)$, and postpartum endometritis $(14.8 \%$ versus $31 \%, p=0.001)$. In crude and adjusted models, when comparing the azithromycin group with the erythromycin group, a decreased risk was noted for the development of clinical chorioamnionitis, neonatal sepsis, and postpartum endometritis. Pregnancy latency by regimen was not significantly different in crude and adjusted models. Conclusion. Our study suggests that latency antibiotic regimens substituting azithromycin for erythromycin have lower rates and decreased risk of clinical chorioamnionitis, neonatal sepsis, and postpartum endometritis with no difference in pregnancy latency.
\end{abstract}

\section{Introduction}

Preterm prelabor rupture of membranes (PPROM) refers to prelabor rupture of membranes before 37 0/7 weeks of gestation. It is responsible for approximately one-third of preterm births and the single most common identifiable factor associated with preterm delivery [1-3]. The management of PPROM remains among the most controversial issues in maternal-fetal medicine. Remaining points of contention include duration and regimen of administration of antibiotic prophylaxis with goals of prolonging pregnancy latency and providing prophylaxis against maternal and fetal infection. The rationale for antibiotic prophylaxis is that infection appears to be both a cause and consequence of PPROM. Infection may lead to spontaneous preterm labor or may be the indication for medically indicated preterm delivery. The importance of reducing infection is highlighted by studies suggesting a relationship between chorioamnionitis, duration of membrane rupture, and development of cerebral palsy [4]. Currently, a regimen with reasonable activity against the 
major pelvic pathogens is employed for prophylaxis, but the optimal regimen is unclear.

The traditional and originally described regimen for prophylaxis of chorioamnionitis and increased pregnancy latency was outlined first by Mercer et al. [5] and involves intravenous ampicillin $2 \mathrm{~g}$ every 6 hours and erythromycin $250 \mathrm{mg}$ every 6 hours for 48 hours followed by oral amoxicillin $250 \mathrm{mg}$ every 8 hours and erythromycin $333 \mathrm{mg}$ every 8 hours for five days. Presently, there are 3 retrospective studies [6-8] investigating the substitution of azithromycin for erythromycin in the setting of PPROM, each with unique conclusions and dosing strategies of each antibiotic: Pierson et al. [6] found no differences in latency from membrane rupture as well as no difference in secondary maternal and neonatal outcomes. Finneran et al. [7] also found no difference in pregnancy latency but noted a higher rate of cesarean delivery and positive neonatal blood cultures in those patients who received erythromycin for prophylaxis. Navathe et al. [8], when looking at patients who received a similar regimen to Pierson et al., Finneran et al., and this present study, found no differences in pregnancy latency, incidence of chorioamnionitis, or neonatal outcomes.

There are presently no prospective studies investigating the substitution of azithromycin for erythromycin in the setting of PPROM. The objective of this study was to determine if substituting azithromycin for erythromycin has an impact on pregnancy latency and development of chorioamnionitis in the context of PPROM.

\section{Materials and Methods}

We conducted a multicenter, prospective observational cohort study from July 2016 to November 2019 and followed all women receiving latency antibiotic treatment involving either azithromycin or erythromycin in the context of PPROM. Choice of regimen was determined by attending physician preference as either antibiotic was available at all sites involved. The azithromycin group consisted of azithromycin $1 \mathrm{~g}$ PO once and ampicillin $2 \mathrm{~g}$ every 6 hours IV for 48 hours followed by 5 days of amoxicillin $250 \mathrm{mg}$ every 8 hours PO for 5 days. The erythromycin group consisted of erythromycin $250 \mathrm{mg}$ and ampicillin $2 \mathrm{~g}$ every 6 hours IV for 48 hours followed by amoxicillin $250 \mathrm{mg}$ and erythromycin 500 mg every 8 hours PO for 5 days.

Primary outcomes were the duration of pregnancy latency period and development of chorioamnionitis via either clinical or histologic criteria. Clinical chorioamnionitis was defined as outlined Higgins et al. [9]: by maternal temperature of $38^{\circ} \mathrm{C}$ or greater, without another source of fever, and with fetal tachycardia (greater than 160 beats per minute). Histological chorioamnionitis was confirmed on placental pathology examination postpartum.

Secondary outcomes included cesarean delivery, meconium-stained amniotic fluid, postpartum endometritis, and neonatal sepsis. Postpartum endometritis was defined as presence of a temperature of $38^{\circ} \mathrm{C}$ or greater, without another source of fever, and presence of fundal tenderness on physical examination in the postpartum period. Neonatal sepsis was defined as having a positive blood culture.
Patients were excluded if latency antibiotic regimen included combinations apart from those defined, if patients were less than 24 weeks gestational age, had been prescribed other antibiotics before presentation, had a cervical cerclage in situ, had a congenital or lethal fetal anomaly, or had a history of trauma or injury resulting in PPROM. All appropriate and required institutional approvals were obtained for this study.

2.1. Statistical Analysis. To account for unequal variance between treatment groups, Welch two-sample two-tailed $t$ -test and Wilcoxon rank-sum test were used to evaluate differences between continuous variables as appropriate [10]. To evaluate differences between frequencies of binary variables between the treatment groups, we employed $X^{2}$ test for comparing contingency tables as appropriate [11]. To examine treatment regimen effect on pregnancy latency, we employed Kaplan-Meier survival analysis [12]. To assess the contribution of each individual covariate on how it may effect the predictive ability of our model, we conducted the univariable Mantel-Cox proportional hazard model [13]. To further estimate the combined effect of all covariates, we performed multivariable Mantel-Cox proportional hazard model analysis. Relative risks of binary pregnancy outcomes (postpartum endometritis, cesarean delivery, chorioamnionitis, and meconium-stained amniotic fluid) between treatment groups (unadjusted and multivariable adjusted) were calculated using "modified Poisson regression". This analysis was also conducted to determine risks of composite intraamniotic infection/inflammation (III) and composite clinical infectious morbidity (CIM). III was defined as the presence of both clinical and/or histologic chorioamnionitis, and CIM was defined as the presence of both clinical chorioamnionitis and/or postpartum endometritis.

According to Zhou et al. [14], "modified Poisson regression" is defined as Poisson regression using robust error variance called sandwich estimation. Adjusted models were controlled for perceived confounding factors including maternal age, advanced maternal age, gestational age at diagnosis of PPROM, nulliparity, BMI, pregestational diabetes, group $\beta$-streptococcus positive (bacteriuria), any hypertensive disorder in pregnancy, and race.

We estimated the sample size a priori. Based upon previous studies [6-8], we estimated that 142 patients in each arm would be required for $99 \%$ power for continuous outcomes and $88 \%$ for binary outcomes to detect a $50 \%$ difference ( $\alpha$ of 0.05 for a two-tailed test). Regarding the primary outcomes, this identified that our population would be powered to detect a noninferiority limit of 3 days of latency and $50 \%$ difference in chorioamnionitis. All statistics were performed using $\mathrm{R}$ version 3.4.0 [15].

\section{Results}

This study included 310 patients, with 142 receiving the azithromycin regimen and 168 receiving the erythromycin regimen (Figure 1). Maternal demographics included maternal age, advanced maternal age (AMA), gestational age at initial diagnosis of PPROM, BMI, pregestational diabetes, group $\beta$ - 


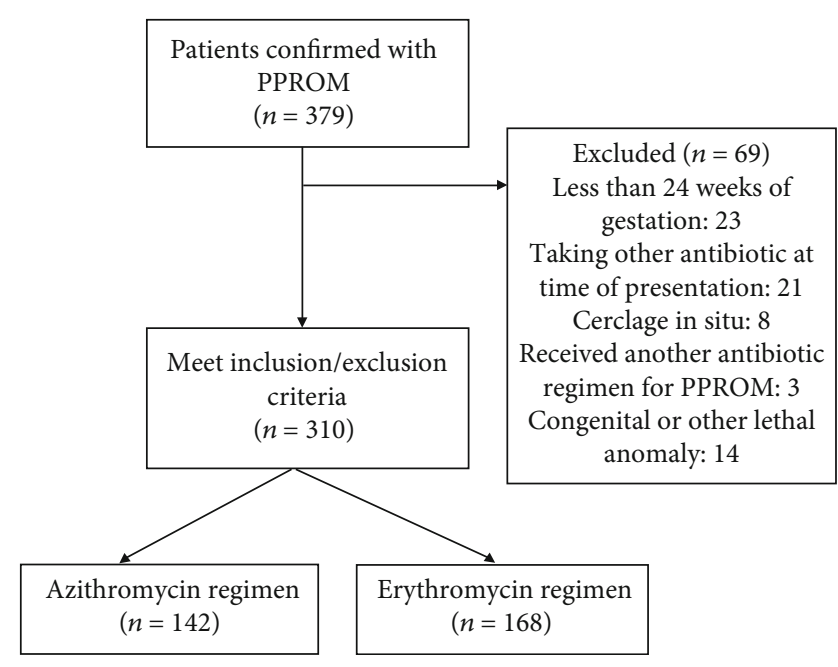

Figure 1: Flow chart of patient selection. 379 patients were confirmed with PPROM. 69 patients were excluded: 23 for being less than 24 weeks of gestation, 21 for taking other antibiotics at time of presentation, 8 with cerclages in situ, 3 who received another antibiotic regimen for PPROM, and 14 with a congenital or lethal anomaly. Of the 310 patients who met the inclusion/exclusion criteria, 142 received azithromycin while 168 received erythromycin.

streptococcus positive (bacteriuria), any hypertensive disorder in pregnancy (including chronic hypertension, gestational hypertension, or preeclampsia with or without severe features), and race. Maternal demographics are shown in Table 1. A statistically significant difference was noted in percentage of nulliparous patients with $X^{2} p$ value of 0.042 with all other demographic components being not significantly different.

Comparison of pregnancy and neonatal outcomes between treatment groups is shown in Table 2. Regarding the primary outcomes, we observed there was no difference in pregnancy latency and significant differences in the rates of clinical chorioamnionitis, but not histologic chorioamnionitis. The difference in the latency period between treatment groups was median of 5 days, interquartile range (IQR) (611 ) days for the azithromycin group versus 4.5 days, IQR (6-10.8) for erythromycin group $(p=0.836$, Wilcoxon Rank-sum Test). Rates of clinical chorioamnionitis were significantly lower in the azithromycin group versus the erythromycin group ( $13.4 \%$ versus $25 \%, p=0.035, X^{2}$ test) while rates of histologic chorioamnionitis were not significantly different between groups (69.7\% in the azithromycin group versus $64.9 \%$ in the erythromycin group, $p=0.367, X^{2}$ test).

Regarding secondary outcomes, statistically significant differences were noted in the rates of postpartum endometritis, neonatal sepsis, and meconium-stained amniotic fluid. No differences were noted in rates of cesarean delivery. The azithromycin group was noted to have lower rates of postpartum of endometritis at $14.8 \%$ versus $31 \%$ in the erythromycin group, $p=<0.001, X^{2}$ test. Rates of meconium-stained amniotic fluid were noted to be higher in the azithromycin group as compared with the erythromycin group at a rate of $26.8 \%$ versus $15.15 \%, p=0.14$. Rates of neonatal sepsis were higher in the azithromycin group as compared with the azithromycin group at a rate of $4.9 \%$ versus $14.9 \%, p=0.004, X^{2}$ test).

Effect of treatment regimens on pregnancy latency using multivariable (adjusted) analysis with the Mantel-Cox proportional hazard model with hazard ratios are shown graphically in Figure 2. This analysis demonstrated that all covariates in isolation did not effect pregnancy latency in the multivariable (adjusted) model. Unadjusted and adjusted Kaplan-Meier survival analyses regarding pregnancy latency are shown in Figures 3 and 4, respectively. This demonstrated no difference in pregnancy latency between treatment types ( $p=0.64$, log-rank test). After controlling for covariates, the adjusted survival curves by treatment type were not noted to be significantly different ( $p=0.90$, log-rank test).

Crude and adjusted risk ratios for binary pregnancy outcomes including chorioamnionitis, cesarean delivery, meconium-stained amniotic fluid, neonatal sepsis, and postpartum endometritis are shown in Table 3. In crude analysis, a $46 \%$ lower risk of clinical chorioamnionitis was noted in the azithromycin group with a $49 \%$ decreased risk after adjusted analysis, $p=0.024$ and 0.015 , respectively. Regarding postpartum endometritis, a $52 \%$ decreased risk was noted for the azithromycin group in crude analysis and a 54\% decreased risk in the adjusted analysis, $p=0.004$ and 0.002 , respectively. Regarding neonatal sepsis, a $67 \%$ decreased risk was noted for the azithromycin group in crude analysis and a $68 \%$ decreased risk in the adjusted analysis, $p=0.010$ and 0.005 , respectively. Regarding meconium-stained amniotic fluid, a $73 \%$ increased risk was noted for the azithromycin group in crude analysis and a 69\% increased risk in the adjusted analysis, $p=0.031$ and 0.042 , respectively. Regarding CIM, a 50\% decreased risk was noted in the azithromycin group in crude analysis and a 52\% decreased risk in adjusted analysis, $p=<0.001$ for both. All other outcomes including histologic chorioamnionitis, III, and cesarean delivery did not achieve a statistically significant difference in either crude or adjusted models.

Given statistical significance of percentage of nulliparous patients between treatment groups, crude and adjusted risk ratios were conducted for this population of patients within treatment groups in the same manner as noted above and are shown in Table 4 . Of the 218 nulliparous patients present in this study, 108 patients received azithromycin while 110 received erythromycin. When comparing these two groups, the significant decreased risks in clinical chorioamnionitis, postpartum endometritis, CIM, and neonatal sepsis were similarly noted as in the entire study population with the exception of meconium-stained amniotic fluid. In crude analysis, a 57\% lower risk of clinical chorioamnionitis was noted in the azithromycin group, with a $61 \%$ decreased risk after adjusted analysis, $p=0.006$ and 0.003 , respectively. Regarding postpartum endometritis, a 50\% decreased risk was noted for the azithromycin group in crude analysis and a $53 \%$ decreased risk in the adjusted analysis, $p=0.012$ and 0.010 , respectively. Regarding neonatal sepsis, an $81 \%$ decreased risk was noted for the azithromycin group in crude analysis and a 93\% decreased risk in the adjusted analysis, $p \leq 0.001$ for both, respectively. Regarding CIM, a 53\% decreased risk was noted in the azithromycin group in 
TABLe 1: Maternal demographics.

\begin{tabular}{|c|c|c|c|}
\hline Characteristic & $\begin{array}{l}\text { Azithromycin group } \\
\qquad n=142\end{array}$ & $\begin{array}{l}\text { Erythromycin group } \\
\qquad n=168\end{array}$ & $p$ \\
\hline Maternal age (years) & $32 \pm 5.3(20-43)$ & $31.5 \pm 5.8(18-47)$ & $0.410^{*}$ \\
\hline Advanced maternal age & $49(35)$ & $45(27)$ & $0.104^{\delta}$ \\
\hline Gestational age at diagnosis & $29.9 \pm 1.76(25-33)$ & $30.2 \pm 1.71(26-33)$ & $0.380^{*}$ \\
\hline Nulliparous & $108(76.1)$ & $110(65.5)$ & $0.042^{\delta}$ \\
\hline BMI $\left(\mathrm{kg} / \mathrm{m}^{2}\right)$ & $32.8 \pm 5.1(21-48.9)$ & $33.86 \pm 4.1(20.8-43.9)$ & $0.282^{*}$ \\
\hline Pregestational diabetes & $15(10.6)$ & $20(11.9)$ & $0.153^{\delta}$ \\
\hline Group $\beta$-streptococcus positive & $28(19.7)$ & $38(22.6)$ & $0.534^{\delta}$ \\
\hline Hypertensive disorders in pregnancy & $29(20.4)$ & $41(24.4)$ & $0.403^{\delta}$ \\
\hline \multicolumn{4}{|l|}{ Race } \\
\hline White & $60(42.3)$ & $74(44)$ & $0.832^{\delta}$ \\
\hline Asian & $30(21.1)$ & $31(18.5)$ & $0.555^{\delta}$ \\
\hline Hispanic & $37(26.7)$ & $51(30.4)$ & $0.486^{\delta}$ \\
\hline Black & $14(9.9)$ & $13(7.1)$ & $0.509^{\delta}$ \\
\hline
\end{tabular}

Data are presented as mean \pm standard deviation (range) or $\mathrm{n}(\%) .{ }^{\delta}$ Statistics performed using $X^{2}$ test. ${ }^{*}$ Statistics performed using Welch two-sample $t$-test.

TABLE 2: Pregnancy and neonatal outcomes.

\begin{tabular}{lccc}
\hline Characteristic & Azithromycin group & Erythromycin group & $n=168$ \\
& $n=142$ & $42(25)$ & 0.010 \\
Clinical chorioamnionitis & $19(13.4)$ & $109(64.9)$ & 0.367 \\
Histological chorioamnionitis & $99(69.7)$ & $4.75(6-10.8)$ & 0.836 \\
Latency interval & $5(6-11)$ & $83(49.4)$ & 0.470 \\
Cesarean delivery & $76(50.7)$ & $26(15.5)$ & 0.014 \\
Meconium-stained amniotic fluid & $38(26.8)$ & $52(31)$ & $<0.001$ \\
Postpartum endometritis & $21(14.8)$ & $25(14.9)$ & 0.004 \\
Neonatal sepsis & $7(4.9)$ & \\
\hline
\end{tabular}

Data are presented as median (interquartile range) or $n(\%) .{ }^{\delta}$ Statistics performed using $X^{2}$ test. ${ }^{*}$ Statistics performed using the Wilcoxon rank-sum test.

crude analysis and a 57\% decreased risk in adjusted analysis, $p \leq 0.001$ for both. All other outcomes including histologic chorioamnionitis, III, and cesarean delivery did not achieve a statistically significant difference in either crude or adjusted models, similar to the results of the entire study population. Therefore, the significant difference in the percentage of nulliparous patients between treatment groups does not affect the overall conclusions in treatment outcomes.

Detailed multivariable analyses for outcomes achieving a statistical significance via modified Poisson linear regression are shown in Supplemental Tables see (available here); all of which did not achieve statistically significant differences. Univariable analysis of the pregnancy outcomes in relation to the factor of nulliparity, given its statistical significance between treatment groups, is shown in Supplemental Tables (available here) and was noted to have a significant effect in composite intra-amniotic infection/inflammation and histologic chorioamnionitis.

\section{Discussion}

This is the first prospective study to evaluate difference in antibiotic regimens substituting azithromycin for erythro- mycin. Our study suggests that latency antibiotic regimens substituting azithromycin for erythromycin have a significantly lower rates and risk of clinical chorioamnionitis, neonatal sepsis, postpartum endometritis, and CIM, with an increased rate and risk of meconium-stained amniotic fluid.

Regarding our primary outcomes, our finding of no differences in pregnancy latency is consistent with the previous studies by Pierson et al., Finneran et al., and Navathe et al. Our results differ from the previous three studies in regards to rates and risk of clinical chorioamnionitis where we found significantly lower rates for the azithromycin versus erythromycin regimens. However, when evaluating for III, our study found no differences between groups, which is consistent with prior studies.

Regarding secondary outcomes, our findings of lower rates of neonatal sepsis is consistent with Finneran et al. but differs from Pierson et al. and Navathe et al. Our results demonstrate rates similar to Finneran et al. in terms of neonatal sepsis via positive blood culture and lower rates when substituting azithromycin for erythromycin. This congruence strengthens the possibility that substitution of azithromycin for erythromycin may provide neonatal benefit. Our findings, both before and after adjusting for confounding 


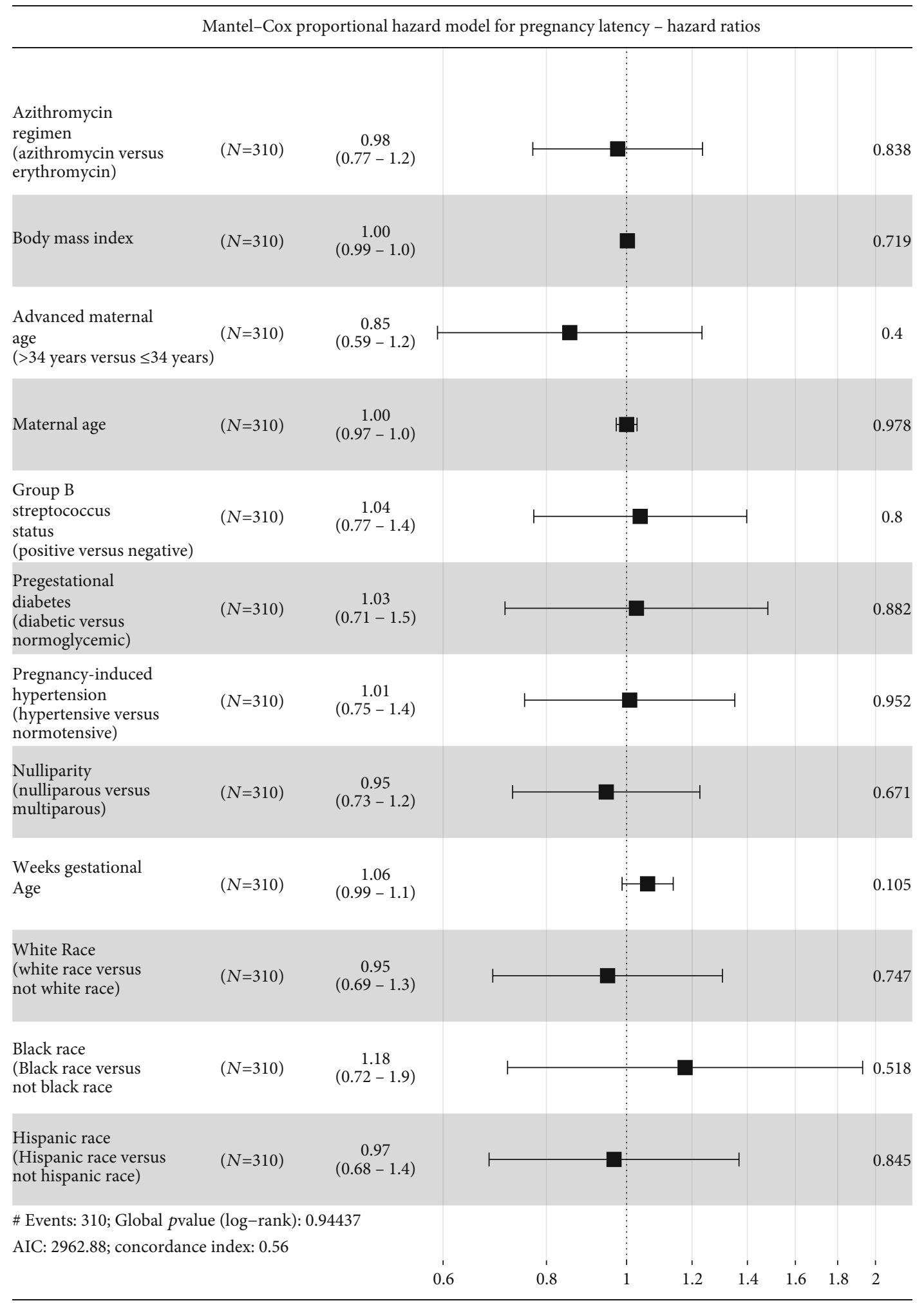

Figure 2: Mantel-Cox proportional hazard model with hazard ratios. This analysis demonstrated that all covariates in isolation did not effect pregnancy latency in the multivariable (adjusted) model.

factors, of a lower rate and risk of clinical chorioamnionitis are consistent with our additional finding of lower rates and risk of postpartum endometritis, likely given the association of both clinical scenarios and indeed is reflected in the significant difference of CIM between the two treatment groups. The finding of increased rates and risk of meconium-stained amniotic fluid is of undetermined significance. 


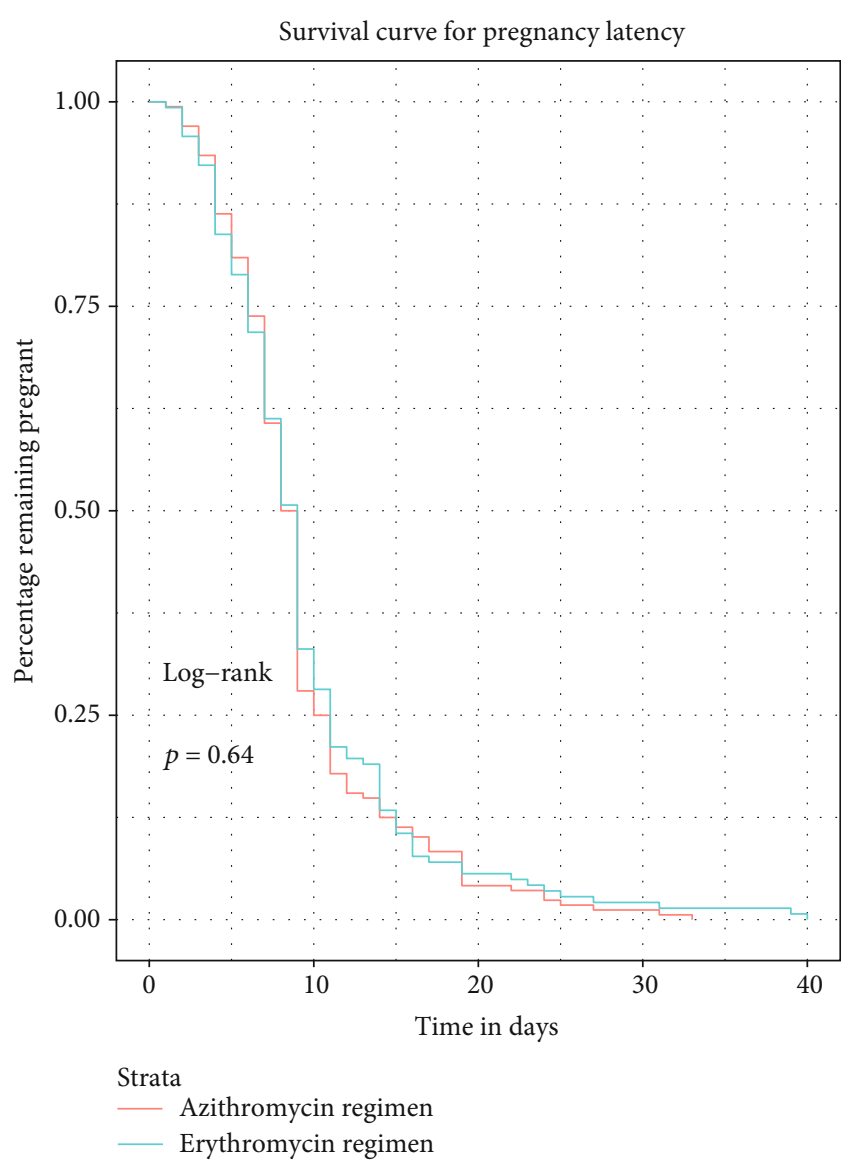

Figure 3: Survival curve for pregnancy latency. This demonstrated no difference in pregnancy latency between treatment types $(p=0.64, \log$-rank test).

We believe these findings to be generalizable across different races and varied geographical settings given that both patient populations were similarly racially diverse. Our populations, however, were predominantly White $(43 \%$ and $42 \%$ ) and Hispanic race ( $36 \%$ and $42 \%$ ), and thus, the results of our study are most applicable to patients of those races. This difference in race may additionally contribute to the differences in results from previous studies where our study had a larger representation of Hispanic race as compared to Pierson et al. who had predominantly White $(48 \%$ and $43 \%)$ and Black (32\% and $32.3 \%)$ race, Finneran et al. who had predominantly Black $(47.6 \%$ and $44.9 \%)$ and White $(28.6 \%$ and $26.9 \%$ ), and Navathe et al. who (in the single-dose azithromycin arm) had predominantly Black race $(43.5 \%$ and $41.0 \%)$.

This study was additionally limited in that the antibiotic regimens were decided solely on physician preference. The preference for latency antibiotic regimen was determined by the covering attending physician at the time of diagnosis. No specific analysis was performed regarding specific physician preference and remains a limitation of this study.

Other regimens employed for the management of PPROM were not considered, which prohibits a more robust analysis of which antibiotic regimen is indeed the most beneficial. Our institution does not use regimens apart from

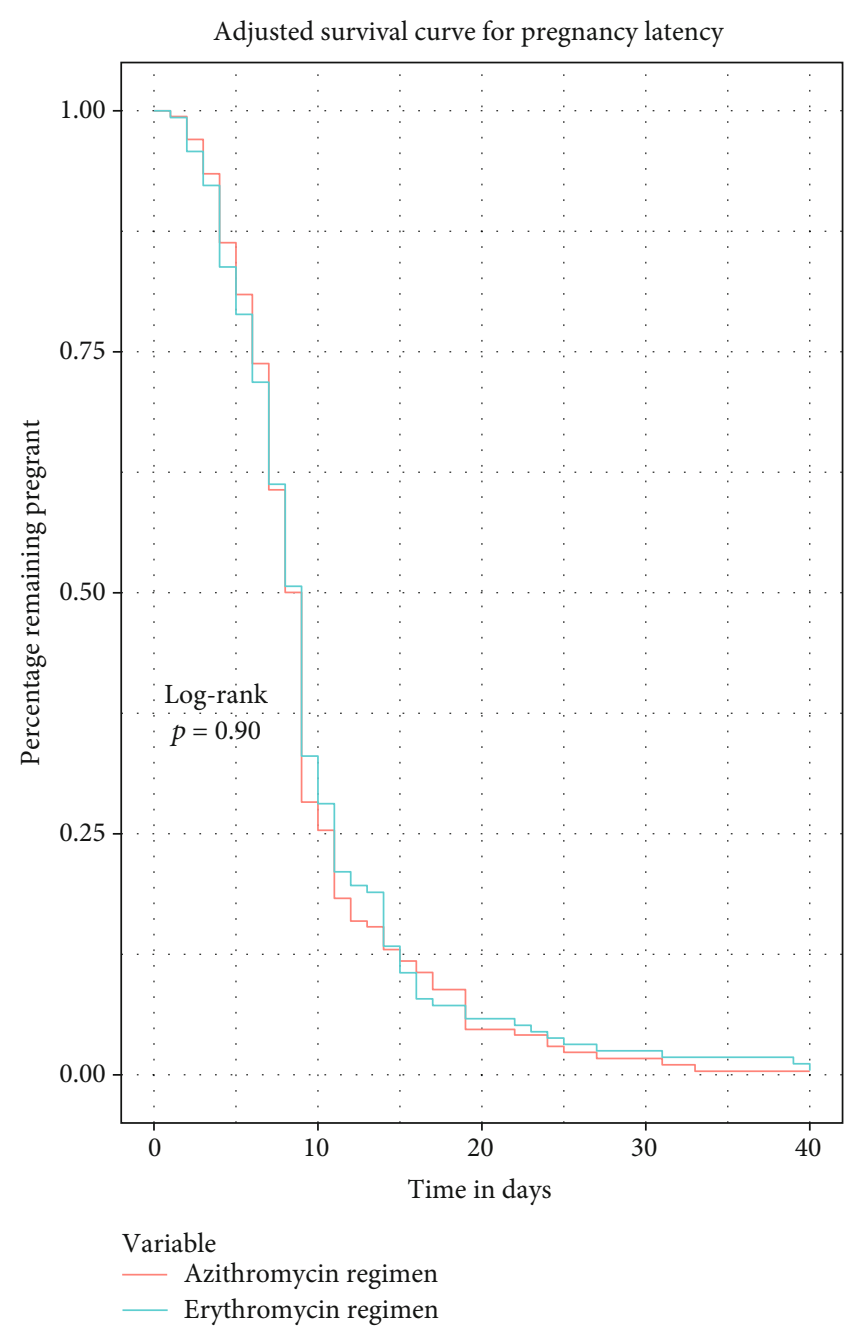

FIgURE 4: Adjusted survival curve for pregnancy latency. After controlling for covariates, the adjusted survival curves by treatment type were not noted to be significantly different $(p=0.90, \log$-rank test $)$.

those included in the study and thus is limited on that basis. Through our robust Kaplan-Meier and Mantel-Cox analysis comparing difference in treatment types and specific covariates within those treatment types, we can conclude with confidence that in this prospective study, the type of antibiotic regimen did not make a difference in pregnancy latency and that this effect was not confounded by other factors, which is in agreement with the conclusions of previous studies that stated there was no difference noted in pregnancy latency.

\section{Conclusion}

Antibiotic regimens substituting azithromycin for erythromycin appear to have no difference in pregnancy latency yet demonstrates significantly lower rates and decreased risk of neonatal sepsis, clinical chorioamnionitis, and postpartum endometritis and an increased risk of meconium-stained amniotic fluid. Further studies are required to elucidate the differences in the findings of this prospective study to that 
TABLE 3: Crude and adjusted risk ratios for pregnancy outcomes for azithromycin versus erythromycin regimens.

\begin{tabular}{|c|c|c|c|c|c|c|}
\hline & \multicolumn{3}{|c|}{ Crude } & \multicolumn{3}{|c|}{ Adjusted $*$} \\
\hline & $\mathrm{RR}$ & $95 \% \mathrm{CI}$ & $p^{+}$ & $\mathrm{RR}$ & $95 \% \mathrm{CI}$ & $p^{+}$ \\
\hline Clinical chorioamnionitis & 0.54 & 0.31 to 0.92 & 0.024 & 0.51 & 0.30 to 0.89 & 0.015 \\
\hline Histologic chorioamnionitis & 1.07 & 0.82 to 1.41 & 0.604 & 1.08 & 0.82 to 1.43 & 0.569 \\
\hline Postpartum endometritis & 0.48 & 0.29 to 0.79 & 0.004 & 0.46 & 0.27 to 0.76 & 0.002 \\
\hline Composite intra-amniotic infection/inflammation & 0.92 & 0.73 to 1.18 & 0.523 & 0.92 & 0.72 to 1.18 & 0.524 \\
\hline Composite clinical infectious morbidity & 0.50 & 0.35 to 0.73 & $<0.001$ & 0.48 & 0.33 to 0.70 & $<0.001$ \\
\hline Neonatal sepsis (positive blood culture) & 0.33 & 0.14 to 0.77 & 0.010 & 0.32 & 0.14 to 0.76 & 0.005 \\
\hline Cesarean delivery & 1.08 & 0.79 to 1.48 & 0.614 & 1.05 & 0.76 to 1.44 & 0.774 \\
\hline Meconium-stained amniotic fluid & 1.73 & 1.05 to 2.85 & 0.031 & 1.69 & 1.01 to 2.81 & 0.042 \\
\hline
\end{tabular}

${ }^{*}$ Models were adjusted for maternal age, advanced maternal age, gestational age at diagnosis of PPROM, nulliparity, BMI, pregestational diabetes, group $\beta$ streptococcus positive (bacteriuria), any hypertensive disorder in pregnancy, and race. ${ }^{+} p$ values calculated using the likelihood ratio test. Estimates are calculated via modified Poisson generalized linear models. $\mathrm{RR}=$ risk ratio; $\mathrm{CI}=$ confidence interval.

TABLE 4: Crude and adjusted risk ratios for pregnancy outcomes for azithromycin versus erythromycin regimens for nulliparous patients.

\begin{tabular}{|c|c|c|c|c|c|c|}
\hline & \multicolumn{3}{|c|}{ Crude } & \multicolumn{3}{|c|}{ Adjusted $*$} \\
\hline & $\mathrm{RR}$ & $95 \% \mathrm{CI}$ & $p^{+}$ & $\mathrm{RR}$ & $95 \% \mathrm{CI}$ & $p^{+}$ \\
\hline Clinical chorioamnionitis & 0.43 & 0.23 to 0.81 & 0.006 & 0.39 & 0.20 to 0.75 & 0.003 \\
\hline Histologic chorioamnionitis & 0.96 & 0.70 to 1.30 & 0.784 & 0.94 & 0.69 to 1.30 & 0.724 \\
\hline Postpartum endometritis & 0.50 & 0.28 to 0.87 & 0.012 & 0.47 & 0.26 to 0.85 & 0.010 \\
\hline Composite intra-amniotic infection/inflammation & 0.81 & 0.62 to 1.06 & 0.127 & 0.78 & 0.59 to 1.04 & 0.092 \\
\hline Composite clinical infectious morbidity & 0.47 & 0.31 to 0.71 & $<0.001$ & 0.43 & 0.28 to 0.67 & $<0.001$ \\
\hline Neonatal sepsis (positive blood culture) & 0.19 & 0.07 to 0.57 & $<0.001$ & 0.20 & 0.07 to 0.59 & $<0.001$ \\
\hline Cesarean delivery & 1.19 & 0.81 to 1.76 & 0.376 & 1.24 & 0.83 to 1.85 & 0.299 \\
\hline Meconium-stained amniotic fluid & 1.48 & 0.84 to 2.61 & 0.176 & 1.40 & 0.78 to 2.52 & 0.263 \\
\hline
\end{tabular}

${ }^{*}$ Models were adjusted for maternal age, advanced maternal age, gestational age at diagnosis of PPROM, nulliparity, BMI, pregestational diabetes, group $\beta$ streptococcus positive (bacteriuria), any hypertensive disorder in pregnancy, and race. ${ }^{+} p$ values calculated using the likelihood ratio test. Estimates are calculated via modified Poisson generalized linear models. $\mathrm{RR}=$ risk ratio; $\mathrm{CI}=$ confidence interval.

of previous retrospective studies. Given that all studies performed do not demonstrate an inferiority of azithromycin substitution over erythromycin, utilizing this substitution remains a reasonable option in the management of PPROM.

\section{Data Availability}

Presently, this data is not available for sharing at this time due to institutional safety and privacy regulations.

\section{Disclosure}

An abstract of this report was presented at the Society of Maternal-Fetal Medicine 39th Annual Pregnancy Meeting on February 14, 2019. (Martingano D, Guan X, Martingano F, Aglialoro G, Singh S. Comparison of antibiotic regimens in the treatment of preterm premature rupture of membranes. American Journal of Obstetrics and Gynecology. 2019 Jan $1 ; 220$ (1).)

\section{Conflicts of Interest}

My coauthors and I have no potential conflicts of interest, real or perceived, and no honorarium, grant, or form of payment was given to anyone to produce the manuscript.

\section{Supplementary Materials}

Supplemental Table 1: multivariable analysis for clinical chorioamnionitis. Supplemental Table 2: multivariable analysis for postpartum endometritis. Supplemental Table 3: multivariable analysis for composite clinical infectious morbidity. Supplemental Table 4: multivariable analysis for neonatal sepsis. Supplemental Table 5: univariable analysis for nulliparity on pregnancy outcomes. (Supplementary Materials)

\section{References}

[1] J. A. Martin, B. E. Hamilton, S. J. Ventura, M. J. Osterman, E. C. Wilson, and T. J. Mathews, "Births: final data for 2010," National Vital Statistics Reports, vol. 61, no. 1, pp. 1-72, 2012.

[2] T. J. Mathews and M. F. MacDorman, "Infant mortality statistics from the 2006 period linked birth/infant death data set," National Vital Statistics Reports, vol. 58, no. 17, pp. 1-31, 2010.

[3] T. P. Waters and B. Mercer, "Preterm PROM: prediction, prevention, principles," Clinical Obstetrics and Gynecology, vol. 54, no. 2, pp. 307-312, 2011.

[4] G. C. Di Renzo, L. C. Roura, F. Facchinetti et al., "Guidelines for the management of spontaneous preterm labor: identification of spontaneous preterm labor, diagnosis of preterm premature rupture of membranes, and preventive tools for 
preterm birth," The Journal of Maternal-Fetal \& Neonatal Medicine, vol. 24, no. 5, pp. 659-667, 2011.

[5] B. M. Mercer, M. Miodovnik, G. R. Thurnau et al., "Antibiotic therapy for reduction of infant morbidity after preterm premature rupture of the membranes: a randomized controlled trial," Journal of the American Medical Association, vol. 278, no. 12, pp. 989-995, 1997.

[6] R. C. Pierson, S. S. Gordon, and D. M. Haas, "A retrospective comparison of antibiotic regimens for preterm premature rupture of membranes," Obstetrics and Gynecology, vol. 124, no. 3, pp. 515-519, 2014.

[7] M. M. Finneran, A. Appiagyei, M. Templin, and H. Mertz, "Comparison of azithromycin versus erythromycin for prolongation of latency in pregnancies complicated by preterm premature rupture of membranes," American Journal of Perinatology, vol. 34, no. 11, pp. 1102-1107, 2017.

[8] R. Navathe, C. N. Schoen, P. Heidari et al., "Azithromycin vs erythromycin for the management of preterm premature rupture of membranes," American Journal of Obstetrics \& Gynecology, vol. 221, no. 2, pp. 144.e1-144.e8, 2019.

[9] R. D. Higgins, G. Saade, R. A. Polin et al., "Evaluation and management of women and newborns with a maternal diagnosis of chorioamnionitis: summary of a workshop," Obstetrics and Gynecology, vol. 127, no. 3, pp. 426-436, 2016.

[10] B. L. Welch, "The significance of the difference between two means when the population variances are unequal," Biometrika, vol. 29, no. 3-4, pp. 350-362, 1938.

[11] G. J. Upton, "Fisher's exact test," Journal of the Royal Statistical Society Series A (Statistics in Society), vol. 155, no. 3, pp. 395402, 1992.

[12] G. Zou, "A modified poisson regression approach to prospective studies with binary data," American Journal of Epidemiology, vol. 159, no. 7, pp. 702-706, 2004.

[13] E. L. Kaplan and P. Meier, "Nonparametric estimation from incomplete observations," Journal of the American Statistical Association, vol. 53, no. 282, pp. 457-481, 1958.

[14] D. Y. Lin and L. J. Wei, "The robust inference for the Cox proportional hazards model," Journal of the American Statistical Association, vol. 84, no. 408, pp. 1074-1078, 1989.

[15] R Development Core Team RF, R: a language and environment for statistical computing, 2014. 